\title{
A Hybrid Model Based on Wavelet Decomposition-Reconstruction in Track Irregularity State Forecasting
}

\author{
Chaolong Jia, ${ }^{1}$ Lili Wei, ${ }^{2}$ Hanning Wang, ${ }^{3}$ and Jiulin Yang ${ }^{4}$ \\ ${ }^{1}$ School of Software Engineering, Chongqing University of Posts and Telecommunications, Chongqing 400065, China \\ ${ }^{2}$ Chongqing Public Security Bureau, Chongqing 401147, China \\ ${ }^{3}$ State Key Laboratory of Rail Traffic Control and Safety, Beijing Jiaotong University, Beijing 100044, China \\ ${ }^{4}$ China National Tendering Center of Mach. \& Elec. Equipment, Beijing 100142, China
}

Correspondence should be addressed to Chaolong Jia; jiachaolong@bjtu.edu.cn

Received 28 June 2014; Accepted 24 October 2014

Academic Editor: Hong Chen

Copyright (C) 2015 Chaolong Jia et al. This is an open access article distributed under the Creative Commons Attribution License, which permits unrestricted use, distribution, and reproduction in any medium, provided the original work is properly cited.

\begin{abstract}
Wavelet is able to adapt to the requirements of time-frequency signal analysis automatically and can focus on any details of the signal and then decompose the function into the representation of a series of simple basis functions. It is of theoretical and practical significance. Therefore, this paper does subdivision on track irregularity time series based on the idea of wavelet decompositionreconstruction and tries to find the best fitting forecast model of detail signal and approximate signal obtained through track irregularity time series wavelet decomposition, respectively. On this ideology, piecewise gray-ARMA recursive based on wavelet decomposition and reconstruction (PG-ARMARWDR) and piecewise ANN-ARMA recursive based on wavelet decomposition and reconstruction (PANN-ARMARWDR) models are proposed. Comparison and analysis of two models have shown that both these models can achieve higher accuracy.
\end{abstract}

\section{Introduction}

If the time series analysis model is divided into two categories; linear and nonlinear model, then in many cases it will be difficult to determine whether a time series model belongs to a linear model or a nonlinear model and then it is also difficult to determine which model should be used in the study. In practice, there is few time series with pure linear or nonlinear feature. There is no single model that can adapt to all situations and solve the problem. In the above case, the concept of hybrid model was proposed.

The basic idea of hybrid forecast model is to use the unique features of each model to capture different data models. Therefore, it is common to use the combination of several models in order to overcome the inadequacy of certain single model and to improve forecast accuracy. Various studies have shown that hybrid model forecasts are often better than single model forecasts. Hybrid model forecast is considered to be a more accurate forecast, which can be described as follows.

Suppose that there are $n$ forecasts, such as $\widehat{y}_{1}(t), \widehat{y}_{2}(t), \ldots, \widehat{y}_{n}(t)$. The general form of hybrid forecast model can be defined as:

$$
\widehat{y}(t)=\sum_{i=1}^{n} w_{i} y_{i}(t)
$$

In the formula, $w_{i}$ is weight assigned to $\hat{y}_{i}(t)$, and the sum of all weights equals 1 .

There are several methods to determine the weight of hybrid forecast. For example, the simplest method is to use a simple average forecast to assign weights, and each forecast will be assigned with equal weight. Equivalent weight (EW) uses the arithmetic mean of each forecast, which is a relatively easy and reliable method. Genre et al. [1] drew similar conclusions to results obtained from simple average 
and complex statistical methods through the combination of expert forecast methods. Armstrong [2] considered that it was very important to use simple methods under great uncertainty. Simple model is easier to be understood than complex models and is less error-prone.

In the specific study, Tseng et al. [3] proposed a fuzzy autoregressive moving average (FARIMA) and took its advantages to overcome the limitations of fuzzy regression and autoregressive moving average model (ARIMA) in time series forecast. Chen and Chung proposed high-order fuzzy time series forecasting method based on a hybrid genetic algorithm [4]. Huarng and Yu combined neural networks and fuzzy time series model to make forecasts [5]. Khashei et al. [6] proposed the basic concepts based on artificial neural networks and fuzzy regression model and time series forecast methods in cases of incomplete data. In their model, artificial neural network is used for preprocessing the original data and to provide the necessary context for the application of fuzzy regression model. Chen [7] combined the study of autoregressive integrated moving average with the study of fuzzy time series (a first or higher order), and studied the adequacy and effectiveness. Yu compared the accuracy of FARIMA and ANNs model in fuzzy time series $[3,8]$.

In the past ten years, a major breakthrough in neural network models is joint modeling with other models. The basic idea of this multimodel approach is to use the unique ability of each component model to better capture different data patterns. Theoretical and empirical results show that the combination of different models can be an effective way to improve the model forecast performance, especially in cases when each model has a very different character in the hybrid model. For example, Goh et al. [9] forecasted drug dissolution by using combination model of Elman neural network. Medeiros and Veiga [10] proposed a time series forecasting system for control by blending smooth transition, regression model, and time-varying parameters neural network. Armano et al. [11] forecasted stock index by using a hybrid neural network model. Zhang [12] proposed ARIMA time series forecast model hybrid with neural network.

Tseng et al. [13] proposed a hybrid forecasting model, which combined the seasonal time series ARIMA (SARIMA) and back-propagation neural network (BPNN) model, named as SARIMABP. Karunasinghe and Liong [14] studied the performance of chaotic time series of artificial neural network model as a global model compared to the widely applicable local model (local averaging techniques and local polynomial technology). Aitkenhead et al. [15] measured hourly oil, water, and climate variables for several months in the North East of Scotland by using data loggers and other measuring devices in both cases in their paper, and three different methods were used to train the neural network, in which a new biological approximation system was included. BuHamra et al. [16] combined with Box-Jenkins (B-J) model and artificial neural network model to model on Kuwait water consumption time series. Niska et al. [17] used artificial neural networks to make nonlinear models on air quality, but due to its chaotic feature, nonlinear phenomena, and high-dimensional sample space, it was destined to be a daunting task. Zhang and Qi [18] proposed a hybrid time series forecast method by combining ARIMA models and neural networks, using the unique advantages of ARIMA models for linear and ANN for nonlinear modeling. Kim [19] used support vector machine (SVM) for financial time series forecast and applied SVM to forecast stock price index in the study. Ho et al. analyzed and compared neural networks and ARIMA modeling in time series modeling, and found that artificial neural network model and BP neural network model can achieve more satisfactory results compared with the ARIMA model. Zhang [20] proposed integration time series forecasting methods of autoregressive integrated moving average and artificial neural network. Ince and Trafalis [21] proposed a two-stage hybrid model, including the employment of parametric technology autoregressive moving average parameter, vector autoregression (VAR) integration, and nonparametric techniques technology methods such as support vector regression (SVR) and artificial neural network forecast exchange rate. Chang et al. [22] studied hybrid model combined with self-organizing map (SOM) neural networks, genetic algorithms (Gas), and fuzzy rule base (FRB) in forecast. Huarng and $\mathrm{Yu}$ [23] used a method combined with description and used neural network to forecast fuzzy time series.

Recently, more and more hybrid forecasting models have been proposed: ARIMA, artificial neural networks (ANNs), and fuzzy logic have shown good performance in the financial time series forecast. Deng and Wang [24] proposed a new incremental learning method (ILA) based on hybrid fuzzy neural network framework. Pai and Lin [25] proposed a hybrid algorithm, using ARIMA models and support vector machines (SVMs) to forecast stock prices. Chen and Wang [26] constructed a seasonal time series forecasting model combined with seasonal autoregressive integrated moving average model (SARIMA) and support vector machine. Zhou and $\mathrm{Hu}$ [27] proposed a hybrid modeling and forecasting based on gray box autoregressive moving average (ARMA) models. Armano et al. [11] proposed a new hybrid approach, integrating genetic algorithms and artificial neural network to forecast the stock market. Yu et al. [28] proposed a new nonlinear forecast model, integrating artificial neural networks and generalized linear autoregression (GLAR) in the foreign exchange market to obtain accurate forecasts. Kim and Shin [29] studied the validity of hybrid method based on neural networks in time series forecast, such as the adaptive time delay neural networks (ATNNs) and time delay neural networks (TDNNs). Zhang [20] proposed hybrid methods by ARIMA and artificial neural network time series forecasting. Tseng et al. [13] proposed SARIMABP hybrid model, combining seasonal ARIMA model (SARIMA) and back propagation (BP) neural network model to forecast seasonal time series data. Khashei et al. [30] proposed a new hybrid model, constituted by the fuzzy logic and artificial neural network structure, in order to get more accurate results as well as to overcome the limitations of ARIMA model. In the first stage, ARIMA model is used for linear time series modeling, and residuals of linear model contain only the nonlinear relationship; in the second stage, the artificial neural network is used to simulate nonlinear model ARIMA model residuals; in the final stage, the model parameters are 
the optimum values calculated by using basic concepts of fuzzy regression, in fuzzy digital form.

Standard deviation of track irregularity reflects the characteristics of track irregularity state change. As a result, this paper takes track irregularities standard deviation as the study object, and makes more comprehensive tendency forecasts about the track irregularity changes. In this study, track irregularity data is provided by State Key Laboratory of Rail Traffic Control and Safety, Beijing Jiaotong University, and K449+000-K450+000 section, Beijing-Kowloon line, from February 20, 2008, to July 23, 2010: 44th cross level inspection data is taken as the study data. The section is divided into 40 units (every $25 \mathrm{~m}$ units as a statistical unit), and the standard deviation of each unit is calculated, and then 40 cross level standard deviation time-series data is obtained.

\section{Traditional ARIMA Model}

ARIMA is one forecast method in time series analysis. In $\operatorname{ARIMA}(p, d, q), \mathrm{AR}$ is autoregression, $p$ is the number of autoregressive term, MA is the moving average, $q$ is the average number of sliding, and $d$ is differential time points to form a smooth sequence, also known as order.

$\operatorname{ARIMA}(p, d, q)$ model is an extension of $\operatorname{ARMA}(p, q)$ model. ARMA models for smooth data directly, with no need for a differential conversion; but ARIMA needs to do first differential to data, and after the differential, time series will be steady, and then modeling process will have the same principles with ARMA. ARIMA $(p, d, q)$ model can be expressed as

$$
\left(1-\sum_{i=1}^{p} \phi_{i} L_{i}\right)(1-L)^{d} X_{t}=\left(1+\sum_{i=1}^{q} \theta_{i} L^{i}\right) \varepsilon_{t} .
$$

In the above model, $L$ is the lag operator, $d \in \mathbb{Z}, d>0$,

The noninterval characteristics of track inspection data cannot be ignored when studying track state change trends. Track irregularity time series is

$$
x^{(0)}=\left(x^{(0)}\left(t_{1}\right), x^{(0)}\left(t_{2}\right), \ldots, x^{(0)}\left(t_{n}\right)\right) .
$$

$x^{(0)}\left(t_{i}\right)$ is the data element of original time series, $t_{i}-t_{i-1} \neq$ constant, $1 \leq i \leq n$.

In time series analysis methods, the first premise is to ensure that the time series data is equipped with equal intervals, and therefore it needs to be converted to equal interval time series. The conversion method that non interval time series convert to equal interval time series:

$$
\begin{aligned}
x_{1}^{(0)}(i)= & x^{(0)}\left(t_{i-1}\right)+\frac{x^{(0)}\left(t_{i}\right)-x^{(0)}\left(t_{i-1}\right)}{t_{i}-t_{i-1}} \\
& \cdot\left(t_{1}+(i-1) \cdot \frac{t_{n}-t_{1}}{n-1}-t_{i-1}\right) .
\end{aligned}
$$

In the formula, $2 \leq i \leq n-1$, and the boundary values in the first and the last of the time series remain unchanged in the conversion process, $x_{1}^{(0)}\left(t_{1}\right)=x^{(0)}\left(t_{1}\right), x_{1}^{(0)}\left(t_{n}\right)=x^{(0)}\left(t_{n}\right)$.

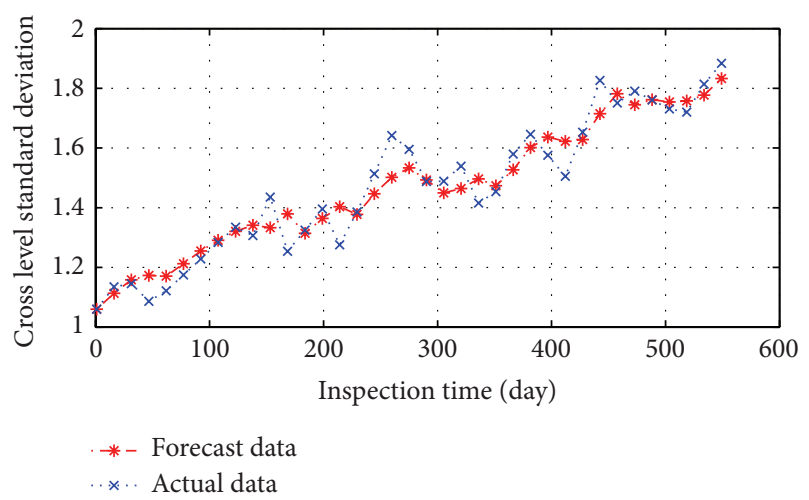

FIGURE 1: Forecast value and the actual value of ARIMA.

ARIMA model forecasted results of 38-cross-level standard deviation data at the K449+800-K449+825 unit sections, from November 13, 2008, to May 18, 2010, after equal interval conversion and the actual data are shown in Figure 1.

From the comparison of forecasted and actual values in Figure 1, the forecasted value is accurate in general, but the accuracy in detail still needs to be improved.

\section{Core Idea of Hybrid Model Based on Wavelet Decomposition-Reconstruction}

The time series can be divided into two parts: low-frequency information and high-frequency information in Wavelet analysis. Low-frequency information is slowly changing part and reflects the changing trend of the time series, and it accounts for the majority of all information; high-frequency information is rapidly changing part (including mutation and jumping) and reflects the details of the time series, and it accounts for a small portion of all information. This is the wavelet decomposition of the first layer. On the basis of the first layer portion, the second layer is decomposed into two parts-the high-frequency information and low-frequency information-and then the third layer is decomposed into a low-frequency information and high-frequency information, and so on. These are single-scale decomposition and the low-frequency part is not redecomposition. If it does multiscale decomposition, the low-frequency part would be decomposed layer by layer like the high-frequency portion.

Wavelet transform is a new transformation analysis, and it inherited and developed the localized ideas of the short-time Fourier transform, while overcoming the shortcoming that the window size does not vary with frequency. It can provide a "time-frequency" window with the frequency change and is the ideal tool for analyzing and processing the timefrequency of signal. Its main feature is that it is able to fully highlight specific aspects of the problem by transform.

Therefore, this paper does subdivision on track irregularity time series based on the idea of wavelet decompositionreconstruction and tries to find the best fitting forecast model of detail signal and approximate signal obtained through track irregularity time series wavelet decomposition, respectively. 


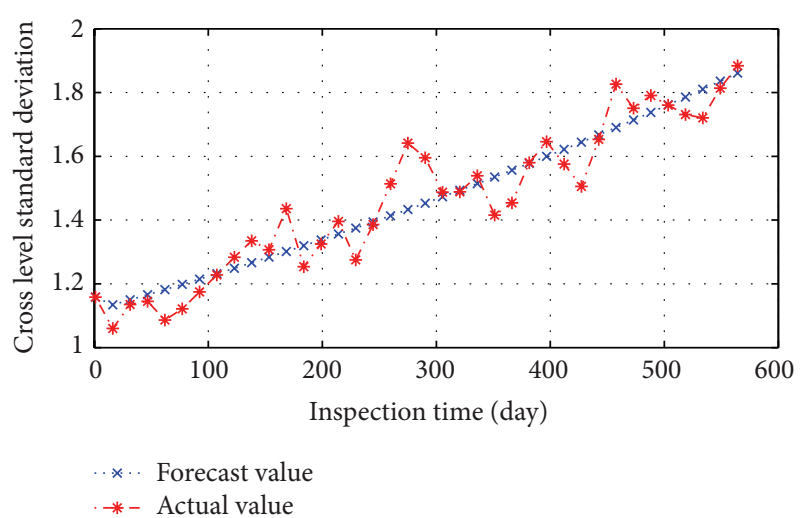

FIGURE 2: The forecast value and the actual value of GM $(1,1)$.

\section{PG-ARMARWDR}

4.1. Traditional GM (1,1) Model. Because of equal interval time series is needed in GM $(1,1)$ forecast, 38-cross-level standard deviation data at the K449+800-K449+825 unit sections, from November 13, 2008, to May 18, 2010, after equal interval transformed is selected for the study. The forecast value and the actual value are shown in Figure 2.

Similarly, from the comparison of the forecast value and the actual value in Figure 2, forecast value has more accuracy in the development direction, but the accuracy in detail still needs to be improved.

\subsection{Improved Model}

4.2.1. Modeling Idea of PG-ARMARWDR. The PLARMARWDR model has achieved better forecast accuracy by using a linear approximation recursive method in lowfrequency approximation waveform signal of cross level standard deviation. More studies indicate that track state changes show exponential trend. As a result, this paper proposes piecewise Gray-ARMA recursive based on wavelet decomposition and reconstruction (PG-ARMARWDR) model.

According to the idea of wavelet decomposition and reconstruction, the standard deviation of track irregularity sequence data waveform signal is decomposed into detail waveform signal $(D 1, D 2$, and $D 3)$ and approximate waveform signal (A3). Among them, detail waveform signal is stationary series with zero mean. The random linear model can be used for study. ARMA model is used in this paper. Similarly, since the interval of track irregularity time series data detection in this study is long, and the sample data are small in scale, so generally the low-frequency approximation waveform signals curve is nonstationary, smooth sequence. Also, some studies have found that track irregularity state cyclical changes showed exponential distribution trends, and the amount of track irregularity change data was small in terms of time, so the piecewise gray recursive model is used in low-frequency approximation waveform signal for trend analysis and to forecast the future state based on historical trends. The modeling idea is shown in Figure 3.
In Figure $3, A 3_{k-4}, A 3_{k-3}, A 3_{k-2}, A 3_{k-1}$, and $A 3_{k}$ are the actual values, $\widehat{A} 3_{k}$ is Piecewise gray forecasted value and $\widehat{A} 3_{k}^{\prime}$ is the forecast value by used piecewise linear approximation recursive model. $A 3_{k-4}, A 3_{k-3}, A 3_{k-2}$, and $A 3_{k-1}$ are used to establish GM $(1,1)$ model and to forecast the future value $A 3_{k}$. Parameter's least squares estimation of models satisfies of the following equation:

$$
\widehat{\alpha}=\left(B^{T} B\right)^{-1} B^{T} Y
$$

In formula (2)

$$
\begin{gathered}
\hat{\alpha}=\left(\begin{array}{l}
\alpha \\
\mu
\end{array}\right), \quad Y=\left(\begin{array}{c}
A 3^{(0)}(2) \\
A 3^{(0)}(3) \\
\vdots \\
A 3^{(0)}(n)
\end{array}\right), \\
B=\left(\begin{array}{cc}
-z^{(1)}(2) & 1 \\
-z^{(1)}(3) & 1 \\
\vdots & \vdots \\
-z^{(1)}(n) & 1
\end{array}\right), \\
z^{(1)}(t)=\frac{1}{2}\left(A 3^{(1)}(t)+A 3^{(1)}(t-1)\right), \quad t=1,2, \ldots, n,
\end{gathered}
$$

$A 3^{(1)}$ refers to one-time accumulated generating sequence (1AGO) of $A 3^{(0)}$.

Solving winterization equation then we can get the response function:

$$
\widehat{A} 3^{(1)}(t)=\left(A 3^{(0)}(1)-\frac{\mu}{\alpha}\right) e^{-\alpha t}+\frac{\mu}{\alpha} .
$$

Formula (7) is equation of GM $(1,1)$ forecast model: the solution of the equation is

$$
\widehat{A} 3^{(1)}(t+1)=\left(\widehat{A} 3^{(1)}(1)-\frac{\mu}{\alpha}\right) e^{-\alpha t}+\frac{\mu}{\alpha}, \quad t=1,2, \ldots, n .
$$

Forecast value $\widehat{A} 3^{(0)}$ is:

$$
\begin{aligned}
\widehat{A} 3^{(0)}(k) & =\beta^{(1)} \widehat{A}^{(1)}(k)=\widehat{A} 3^{(1)}(k)-\widehat{A} 3^{(1)}(k-1) \\
& =\left(1-e^{\alpha}\right)\left(A 3^{(0)}(1)-\frac{\mu}{\alpha}\right) e^{-\alpha(k-1)}
\end{aligned}
$$

Compared with forecast value $\widehat{A} 3_{k}^{\prime}$ by using piecewise linear approximation recursive model in Figure $2, \widehat{A} 3_{k}$ is more close to the actual value.

The forecast value of low-frequency approximation and high-frequency detail time series data signal are added up by weight 1 . The final model is shown in (10)

$$
\widehat{S}=\widehat{D} 1+\widehat{D} 2+\widehat{D} 3+\widehat{A}^{(0)} \text {. }
$$

In the model, $\widehat{D} 1, \widehat{D} 2$, and $\widehat{D} 3$ are high-frequency detail time series signal forecasted by the ARMA model, $\widehat{A} 3$ is time series of the low-frequency approximation by GM $(1,1)$ model forecast, and $\widehat{S}$ is forecast value of the final state.

The modeling process of PG-ARMARWDR is shown in Figure 4 . 


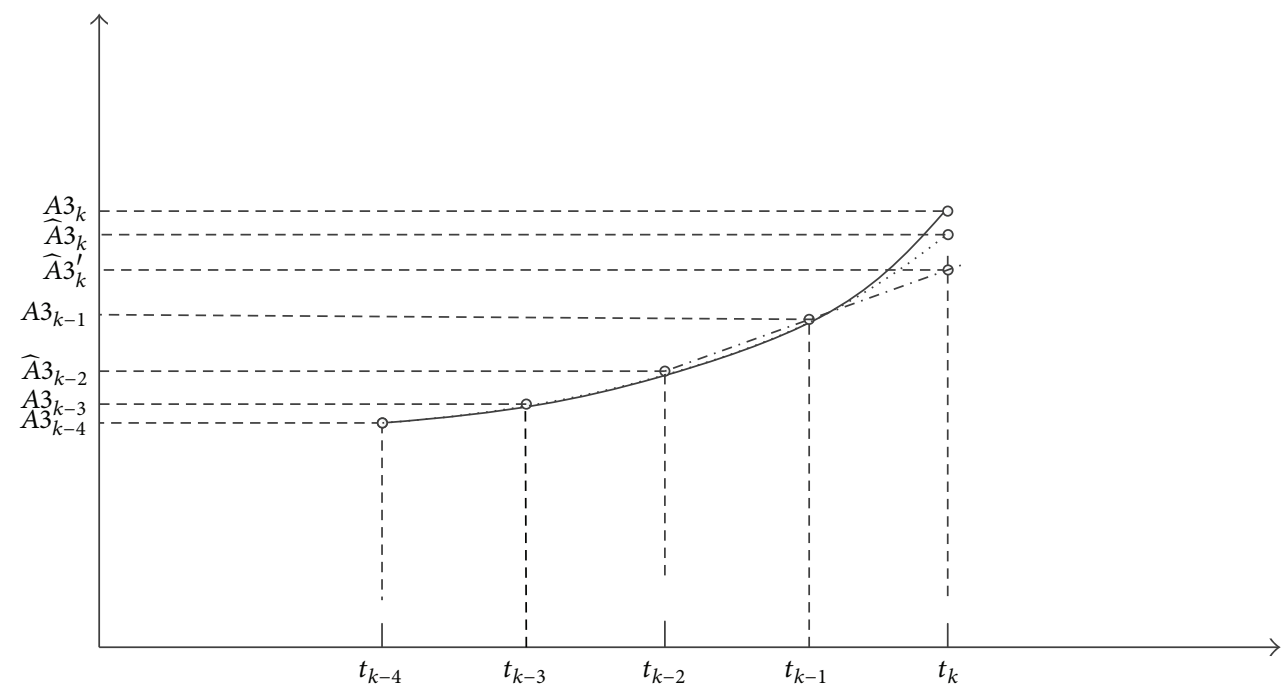

FIgURE 3: Piecewise GM $(1,1)$ approximation recursive forecast model.

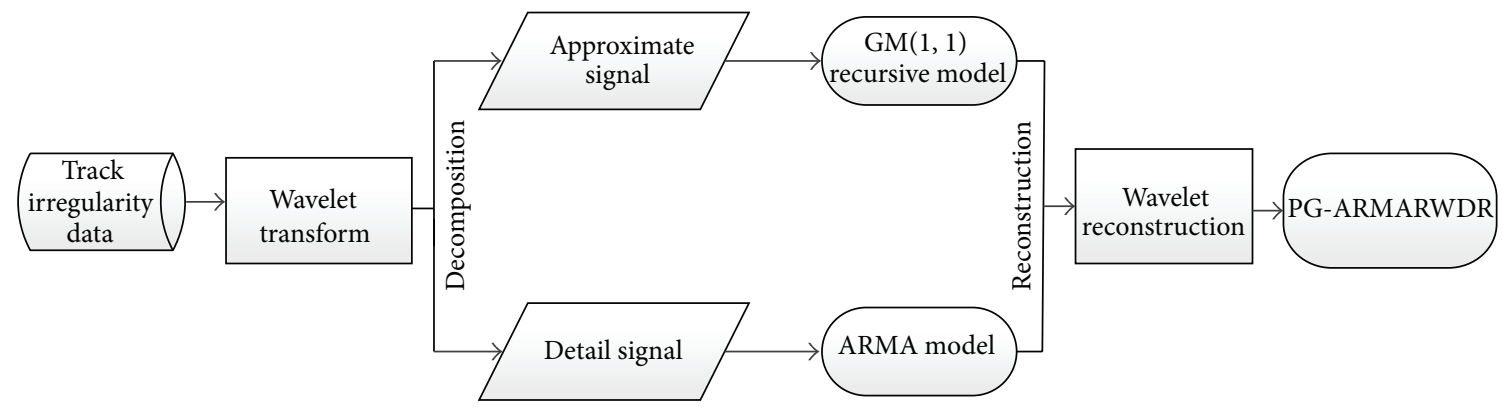

FIGURE 4: Modeling process of PG-ARMARWDR.

4.2.2. Case Study of PG-ARMARWDR. The Daubechies wavelet is used in the decomposition of track irregularity standard deviation time series data signal and the decomposition depth is 3. Decomposition and reconstruction algorithm is Mallat tower algorithm. As for selecting data, track irregularity standard deviation series data trend is taken as the object to make forecast.

Take k449+800-k449+825 unit sections, from November 13, 2008, to May 18, 2010, 38-cross-level standard deviation data after equal time intervals conversion as study data; the PG-ARMARWDR modeling process based on wavelet decomposition and reconstruction is as follows.

(1) Wavelet Decomposition. After wavelet decomposition, cross level standard deviation time series is divided into detail waveform signal in high frequency and approximate waveform signal in low frequency. The decomposition waveform signals of cross level standard deviation time series are shown in Figure 5.

(2) High-Frequency Signals Forecast Model. High-frequency signal after decomposition is stationary time series. ARMA model is used to forecast, and the results are shown in Figures 6,7 , and 8 .
(3) Low-Frequency Signal Piecewise GM (1,1) Recursive Model Forecast. Low-frequency approximation sequence signal after wavelet decomposition is a smooth curve. Piecewise GM $(1,1)$ recursive model is used to make forecast, and recursive forecast results are shown in Table 1.

Forecast curve is shown in Figure 9.

All forecast sequence data is added up by weight 1 . The comparison of forecast values and original values is shown in Figure 10.

4.3. Accuracy and Error. Forecast error of the model is shown in Figure 11.

Accuracy of the model can be measured by MSE and MAPE, and the forecast accuracy of some units is shown in Table 2.

According to the forecast results of MSE, MAPE value in Table 2, PG-ARMARWDR has higher forecast accuracy. This shows that PG-ARMARWDR is an effective way to forecast the trend of track state changes.

\section{PANN-ARMARWDR}

5.1. Artificial Neural Network. Artificial neural networks originally were first proposed by psychologists and mathematical logicians. W. S. McCulloch and W. Pitts from the 

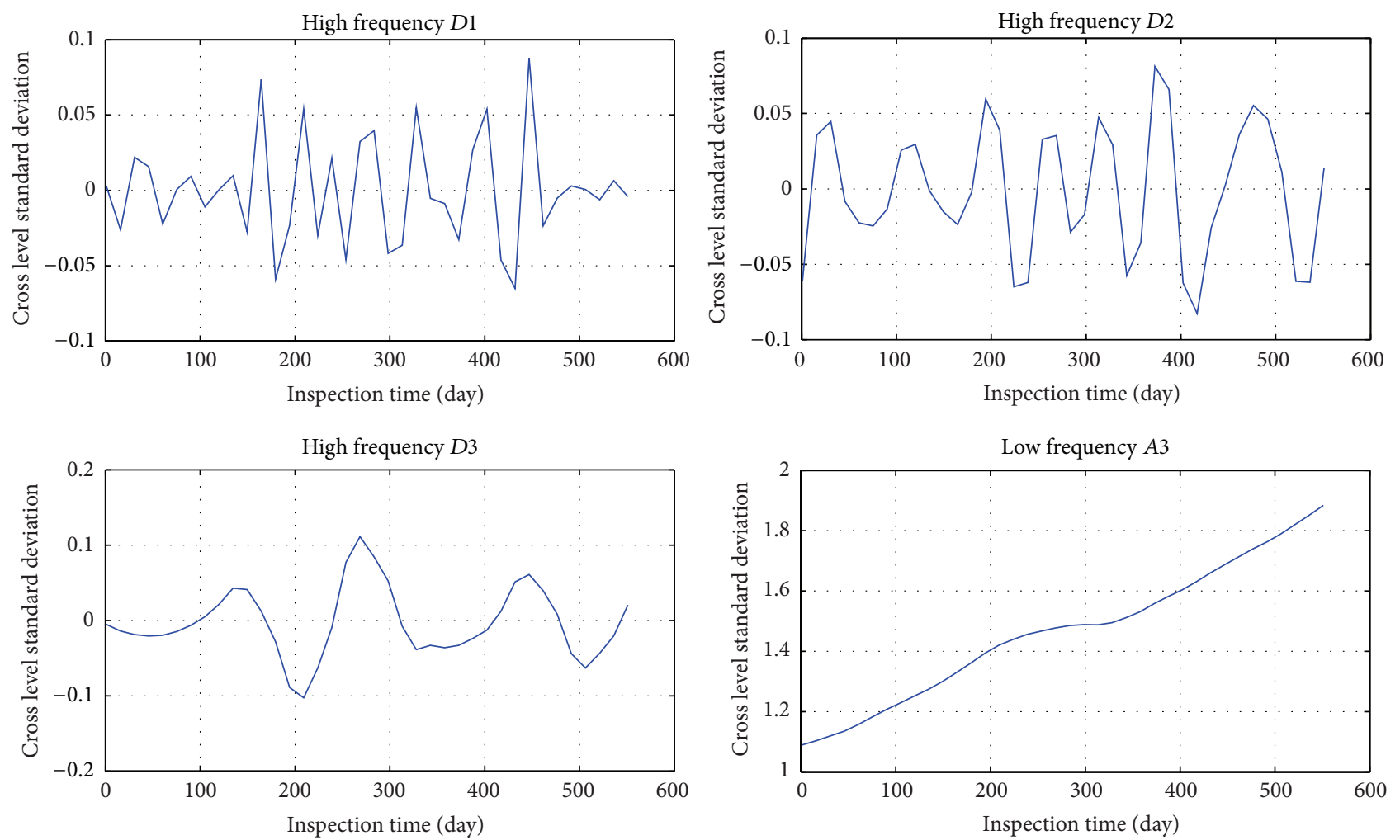

Figure 5: The decomposition waveform signals of cross level standard deviation time series in 3 layers.

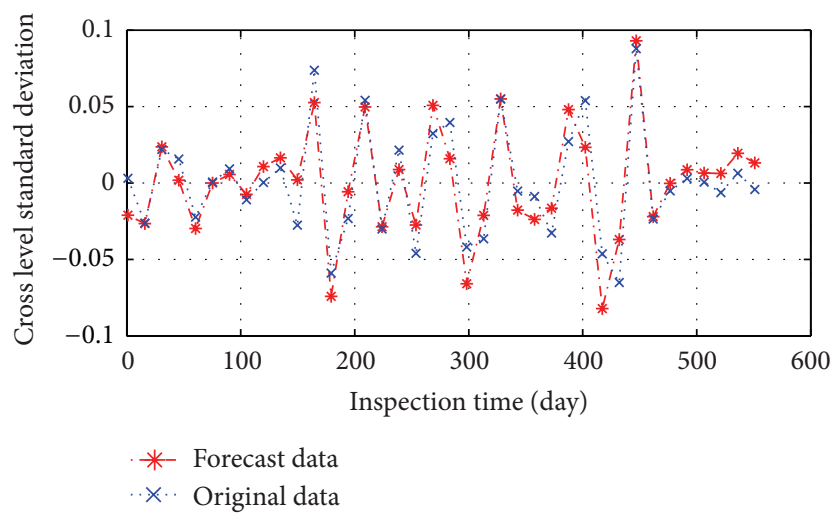

FIGURE 6: Forecast value and the original data of the first layer detail waveform signal.

University of Chicago, pioneer of artificial neural networks, established MP model in 1943, which is the initial neural network and mathematical model. MP model presented a formal mathematical description and network structure methods about neurons, and proved that individual neurons can perform logic functions. Psychologists proposed the idea of variable intensity of synaptic connections in 1949.

The strong nonlinear mapping ability of neural networks can accurately approximate any function through training $[31,32]$. In the mean time, it also has better robustness and can distinguish samples which contain noise [32]. Artificial neural networks can imitate animals' neural networks

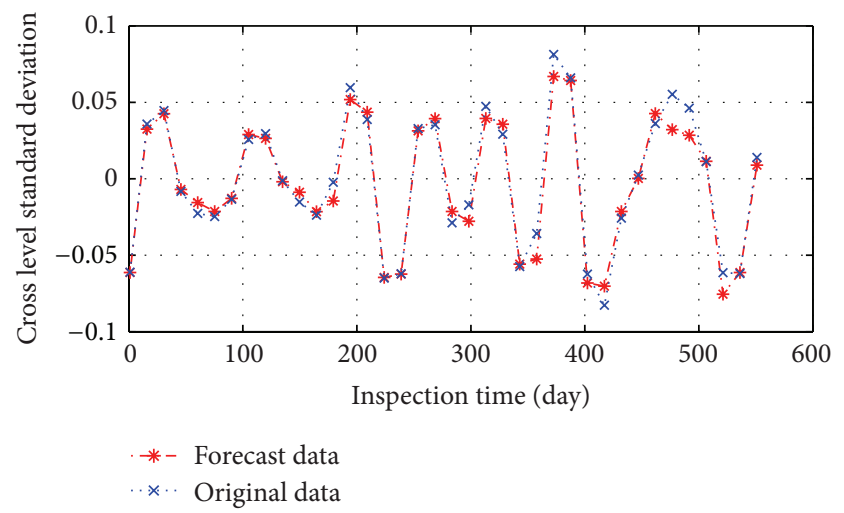

FIGURE 7: Forecast value and the original data of the second layer detail waveform signal.

behavior, and start distributed parallel information processing. It is a mathematical modeling approach with the ability of realizing any complex nonlinear mapping through learning. Artificial neural network relies on system's complexity and can achieve the purpose of processing information by adjusting the internal connection between a large number of nodes. Artificial neural networks have the ability of self-learning and adaptation. They can analyze and master potential laws between a group of mutually corresponding input-output data previously provided and ultimately derive the output with the new input data based on these laws. This study process is called as "training." 
TABLE 1: Forecast formula of low frequency approximation sequence GM $(1,1)$ recursive model.

\begin{tabular}{|c|c|}
\hline $\begin{array}{l}\text { Inspection } \\
\text { serial } \\
\text { number }\end{array}$ & $\begin{array}{l}\text { Forecast formula of low frequency approximation } \\
\text { sequence GM }(1,1) \text { recursive model }\end{array}$ \\
\hline 5 & $\widehat{A} 3^{(0)}(k)=69.251 \exp (0.015786 k)-68.1623$ \\
\hline 6 & $\widehat{A} 3^{(0)}(k)=59.3577 \exp (0.018634 k)-58.2546$ \\
\hline 7 & $\widehat{A} 3^{(0)}(k)=54.6838 \exp (0.020535 k)-53.5651$ \\
\hline 8 & $\widehat{A} 3^{(0)}(k)=56.928 \exp (0.020137 k)-55.7927$ \\
\hline 9 & $\widehat{A 3}{ }^{(0)}(k)=62.0052 \exp (0.018904 k)-60.8484$ \\
\hline 10 & $\widehat{A} 3^{(0)}(k)=65.8802 \exp (0.018153 k)-64.6975$ \\
\hline 11 & $\widehat{A 3}{ }^{(0)}(k)=65.2848 \exp (0.018645 k)-64.078$ \\
\hline 12 & $\widehat{A} 3^{(0)}(k)=61.0115 \exp (0.020284 k)-59.7824$ \\
\hline 13 & $\widehat{A} 3^{(0)}(k)=56.7908 \exp (0.022168 k)-55.5388$ \\
\hline 14 & $\widehat{A} 3^{(0)}(k)=55.3752 \exp (0.023203 k)-54.1011$ \\
\hline 15 & $\widehat{A} 3^{(0)}(k)=59.8461 \exp (0.022003 k)-58.5459$ \\
\hline 16 & $\widehat{A} 3^{(0)}(k)=72.6158 \exp (0.018622 k)-71.2854$ \\
\hline 17 & $\widehat{A} 3^{(0)}(k)=96.3261 \exp (0.014398 k)-94.9648$ \\
\hline 18 & $\widehat{A} 3^{(0)}(k)=130.4074 \exp (0.010849 k)-129.0134$ \\
\hline 19 & $\widehat{A 3}{ }^{(0)}(k)=170.0227 \exp (0.0084436 k)-168.6022$ \\
\hline 20 & $\widehat{A 3}{ }^{(0)}(k)=217.6007 \exp (0.006673 k)-216.161$ \\
\hline 21 & $\widehat{A} 3^{(0)}(k)=296.9763 \exp (0.0049338 k)-295.5202$ \\
\hline 22 & $\widehat{A} 3^{(0)}(k)=638.3549 \exp (0.0023153 k)-636.8875$ \\
\hline 23 & $\widehat{A} 3^{(0)}(k)=847.3693 \exp (0.0017513 k)-845.8921$ \\
\hline 24 & $\widehat{A}^{(0)}(k)=296.2385 \exp (0.0049985 k)-294.7529$ \\
\hline 25 & $\widehat{A} 3^{(0)}(k)=150.6421 \exp (0.0098039 k)-149.1532$ \\
\hline 26 & $\widehat{A} 3^{(0)}(k)=108.1025 \exp (0.013712 k)-106.615$ \\
\hline 27 & $\widehat{A} 3^{(0)}(k)=96.7141 \exp (0.015491 k)-95.2193$ \\
\hline 28 & $\widehat{A 3}{ }^{(0)}(k)=98.1871 \exp (0.015488 k)-96.6757$ \\
\hline 29 & $\widehat{A 3}{ }^{(0)}(k)=102.5414 \exp (0.015077 k)-101.0102$ \\
\hline 30 & $\widehat{A} 3^{(0)}(k)=97.7195 \exp (0.016044 k)-96.1617$ \\
\hline 31 & $\widehat{A} 3^{(0)}(k)=93.688 \exp (0.016974 k)-92.1058$ \\
\hline 32 & $\widehat{A} 3^{(0)}(k)=98.1916 \exp (0.016481 k)-96.5875$ \\
\hline 33 & $\widehat{A 3}{ }^{(0)}(k)=106.5326 \exp (0.015468 k)-104.902$ \\
\hline 34 & $\widehat{A} 3^{(0)}(k)=114.5691 \exp (0.014626 k)-112.9091$ \\
\hline 35 & $\widehat{A} 3^{(0)}(k)=116.9581 \exp (0.014543 k)-115.2707$ \\
\hline 36 & $\widehat{A} 3^{(0)}(k)=112.2606 \exp (0.015359 k)-110.5472$ \\
\hline 37 & $\widehat{A 3}{ }^{(0)}(k)=106.4489 \exp (0.016418 k)-104.7099$ \\
\hline 38 & $\widehat{A 3}{ }^{(0)}(k)=104.752 \exp (0.016945 k)-102.989$ \\
\hline
\end{tabular}

Multi-input and single-output neuron model in neural network structure is shown in Figure 12.

Neuron model can be described with mathematical relationships:

$$
\begin{gathered}
S_{j}=\sum_{i=1}^{n} W_{i j} X_{i}-\theta_{j}, \\
Y_{j}=f\left(S_{j}\right) .
\end{gathered}
$$

In the formula, $X_{i}$ is the input signal, $W_{j i}$ is the connection weights between neurons $j i, f$ is activation function, and activation function must be derivable everywhere.
TABLE 2: Analysis of model accuracy (cross level standard deviation).

\begin{tabular}{lcc}
\hline Unit section & MSE & MAPE \\
\hline K449+000-K449+025 & 0.024 & $2.93 \%$ \\
K449+100-K449+125 & 0.016 & $1.43 \%$ \\
K449+200-K449+225 & 0.028 & $1.77 \%$ \\
K449+325-K449+350 & 0.022 & $1.83 \%$ \\
K449+425-K449+450 & 0.022 & $1.72 \%$ \\
K449+475-K449+500 & 0.023 & $2.40 \%$ \\
K449+575-K449+600 & 0.023 & $1.83 \%$ \\
K449+675-K449+700 & 0.025 & $1.03 \%$ \\
K449+800-K449+825 & 0.017 & $0.84 \%$ \\
K449+875-K449+900 & 0.015 & $1.12 \%$ \\
K449+975-K450+000 & 0.015 & $1.55 \%$ \\
\hline
\end{tabular}

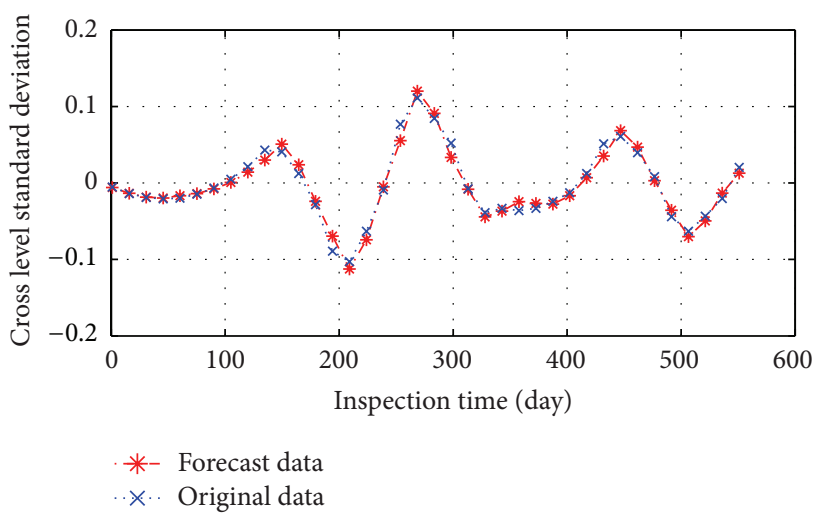

FIGURE 8: Forecast value and the original data of the third layer detail waveform signal.

Artificial neural network (ANN) $[33,34]$ is a powerful tool for handling nonlinear problems, and the accuracy is very high, which is an unparalleled advantage for other methods. That is why it has attracted the scientific community to employ and improve the algorithms [35-38]. BP model $[39,40]$ and RBF model are used widely in neural network model. Rumelhart and McClelland raised network error Back propagation learning algorithm in 1985 [41].

BP neural network is a network that uses back propagation for training. Usually, the network has an input layer an output layer and a hidden (middle) layer. BP algorithm is a training algorithm of acyclic multistage network, whose learning process is composed of forward direction propagation and reverse propagation. The input layer is processed by nonlinear transformation through the input layer to the hidden layer and transmitted to the output layer. The state of neurons at each layer affects the next layer. If the output layer cannot get the desired output, it will be transferred to back propagation, and by modifying the weights of the neuron, the error signal is minimized. BP neural network uses the error of output to estimate the error of the layer that leads directly to the output layer and then estimates errors of more previous layers. Through this layer-to-layer backpropagation, the error estimates of all the other layers can be obtained. 


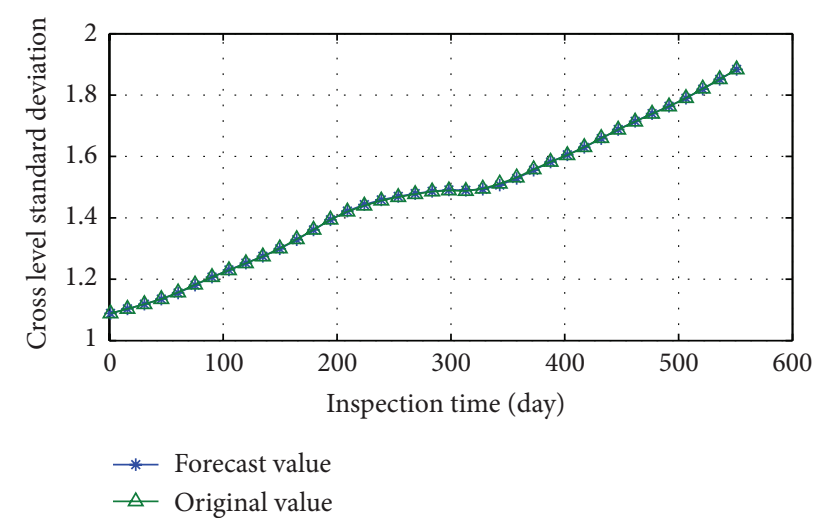

FIgURE 9: Forecast value and the original data of the third layer approximation waveform signal.

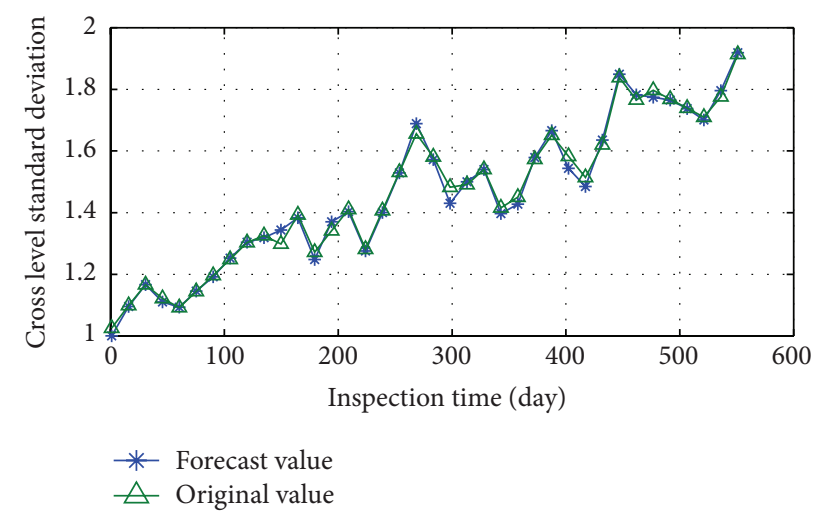

FIGURE 10: Comparison between forecast value and original value.

The guiding principle of BP neural network learning rule is as follows: the correction of network's weights and thresholds should follow after the fastest decline direction of the performance function, negative gradient direction, and the mathematical expression is as follows:

$$
x_{k+1}=x_{k}-a_{k} g_{k} .
$$

In the formula, $x_{k}$ is the current weight and threshold matrix, $g_{k}$ is the gradient of the current performance functions, and $a_{k}$ is the learning rate.

The typical structure of BP neural network model is shown in Figure 13; the output of the hidden layer is weights multiplied by the input value, and then the activation function is substituted; the final output is multiplication and summation of the output of the hidden layer and weights of the hidden layer to the output layer. Among them, there are $n$ neurons in the input layer, $p$ neurons in the hidden layer, and $q$ neurons in the output layer.

5.2. BP Neural Network Track Irregularity State Forecast. Take k449+800-k449+825 unit sections, from November 13, 2008, to May 18, 2010, 38-cross-level standard deviation data after equal time intervals conversion as the object data for study. In specific studies, track cross level standard deviation change time series data of $\mathrm{K} 229+825$ to $\mathrm{K} 449+800$ unit section

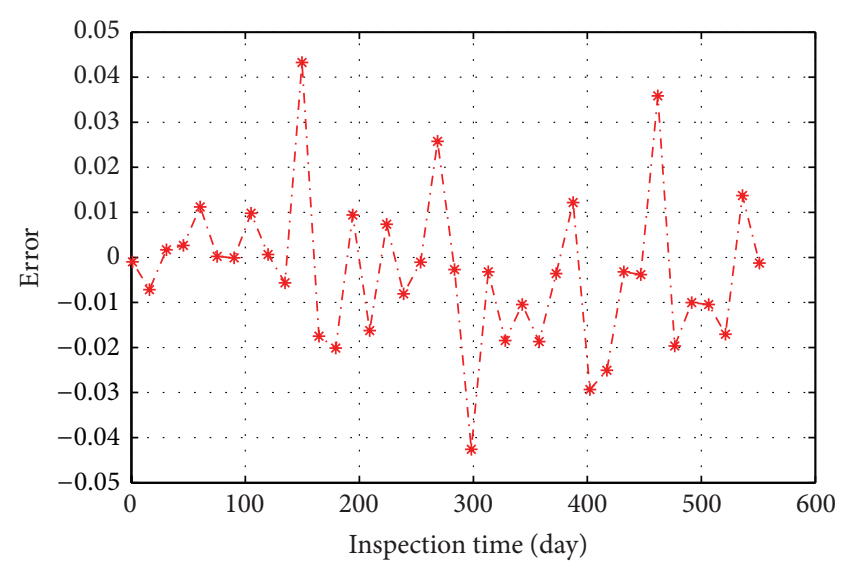

FIGURE 11: Error analysis.

is selected, and model training is based on the following process:previous four track irregularity standard deviation data as the input data and the fifth track irregularity standard deviation data as the output data. The network structure which is composed of four neurons in the input layer and six neurons nodes in the hidden layer and four neurons nodes in the output layer is selected. $S$-function is selected as transfer function of the hidden layer; linear function is selected as transfer function of the output layer. Learning rate is set to be 0.1 , momentum coefficient is set to be 0.95 , and the maximum training time is set to be 30 seconds; variable learning rate momentum gradient descent algorithm (traingdx) is selected as the training algorithm, and minimum mean square error is set to be 0.001 . According to the model, the forecast trend curve resulted from the recursive neural network forecast and the actual curves are shown in Figure 14.

Error analysis of the model is shown in Figure 15.

According to the comparison of forecast values and actual values, forecast values have better accuracy when it is with a smooth development trend, but the forecast error is larger in the presence of mutations. As a result, the methods for application need to be improved to accommodate different situations.

\subsection{Improved Model}

5.3.1. Modeling Idea of PANN-ARMARWDR. The lowfrequency approximation signal after wavelet decomposition which employs a linear model and a gray recursive model has achieved better forecast accuracy in models of PG-ARMARWDR. As an important nonlinear modeling method, neural network model has been widely used. Thus, this section proposes piecewise ANN-ARMA recursive based on wavelet decomposition and reconstruction (PANNARMARWDR) model.

According to the idea of wavelet decomposition and reconstruction, the standard deviation of track irregularity sequence data waveform signal is decomposed into detail waveform signal $(D 1, D 2$, and $D 3)$ and approximate waveform signal ( $A 3)$, in which detail waveform signal is stationary series with zero mean. The random linear model can 


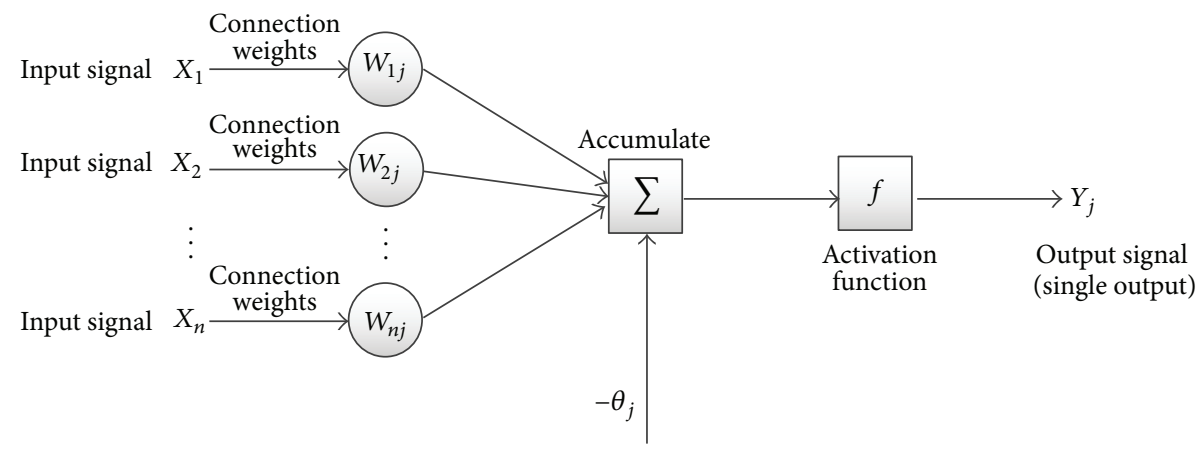

Figure 12: Neuron model.

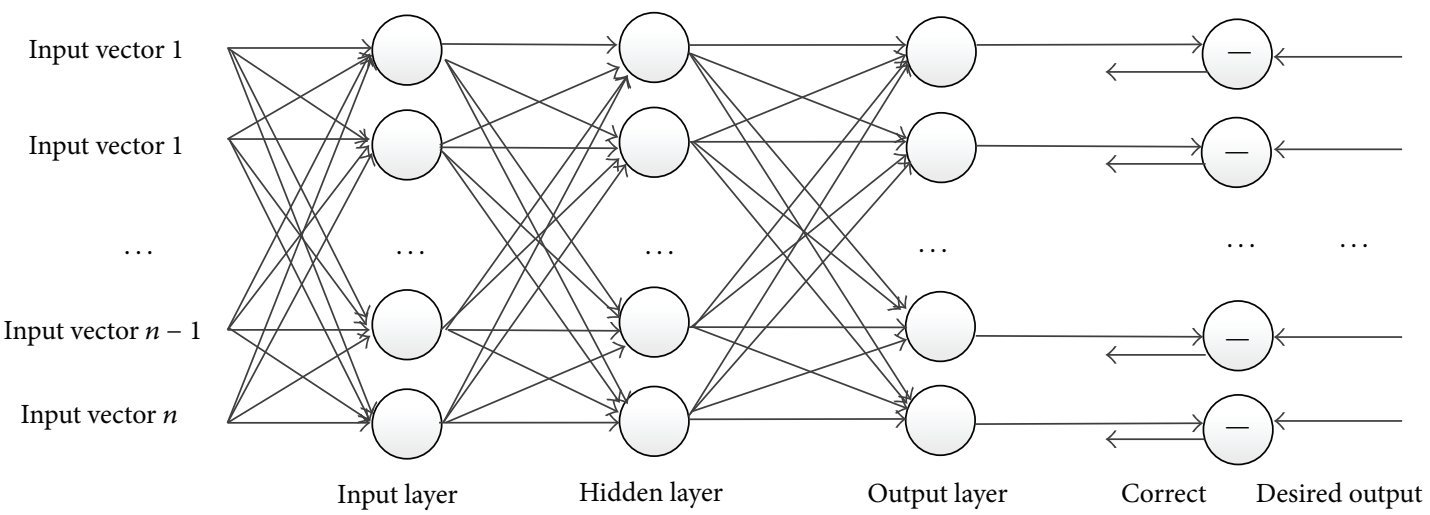

FIGURE 13: Typical BP neural network model.

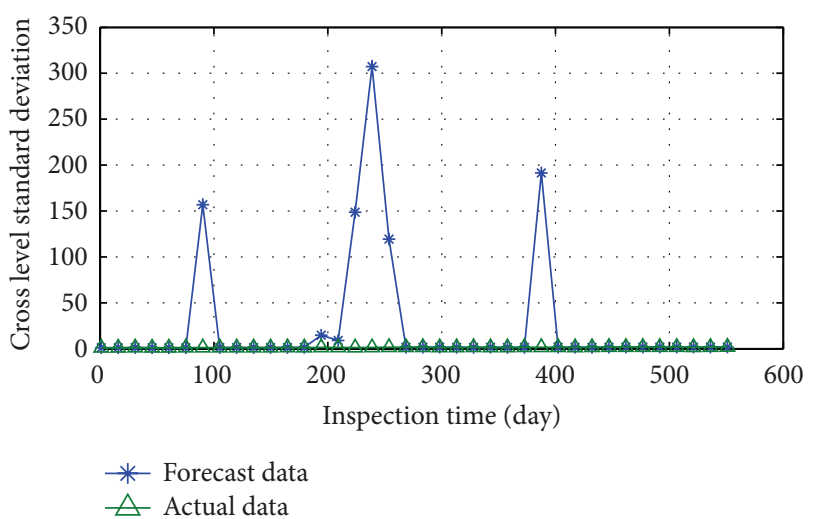

FIGURE 14: Fitting diagram of the actual curve and trend curve of BP ANN model.

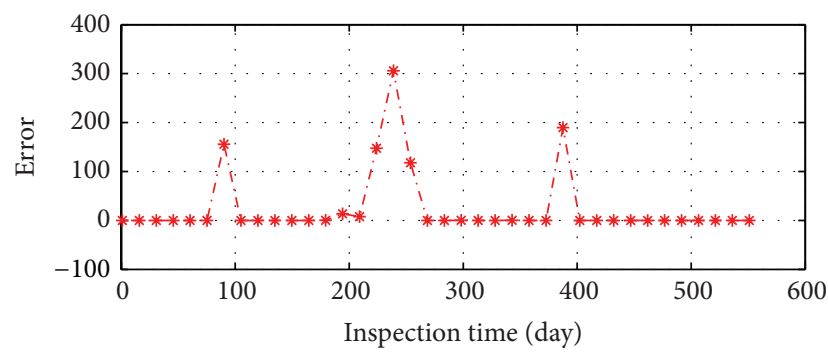

FIGURE 15: Error analysis. be used for research. ARMA model is used in this paper. Similarly, since the interval of track irregularity time series data detection in this study is long, and the sample data are small in scale, so generally the low-frequency approximation waveform signals curve is nonstationary, smooth sequence. In this paper, the ANN model is used in low-frequency approximation waveform signal for trend analysis and to forecast the future state based on historical trends.

In the neural network modeling, inspection data can be seen as input, and the determination result can be regarded as the output, and there is a nonlinear mapping between the input and output.

Triple error back propagation neural network is selected in track state forecast, which is a fully connected network of neurons, namely, neurons achieve full connection between layers and neurons at the same layer are unconnected. Input data is four consecutive data of the track irregularity time series data, and output data is forecast value of the fifth time. The number of neurons nodes in the output layer is the same with the output volume, and the hidden layer nodes are calculated based on experience formula $L=\sqrt{n+m}+a$. In this paper, $L$ is taken as 6 . Each hidden layer neuron has an additional input, thus each hidden layer has six weights, all of which are set to be 0.5 , and the weight is learned in the process of back propagation.

After analysis and calculation, the network structure which is composed of four neurons in the input layer and six neurons nodes in the hidden layer and one neurons 


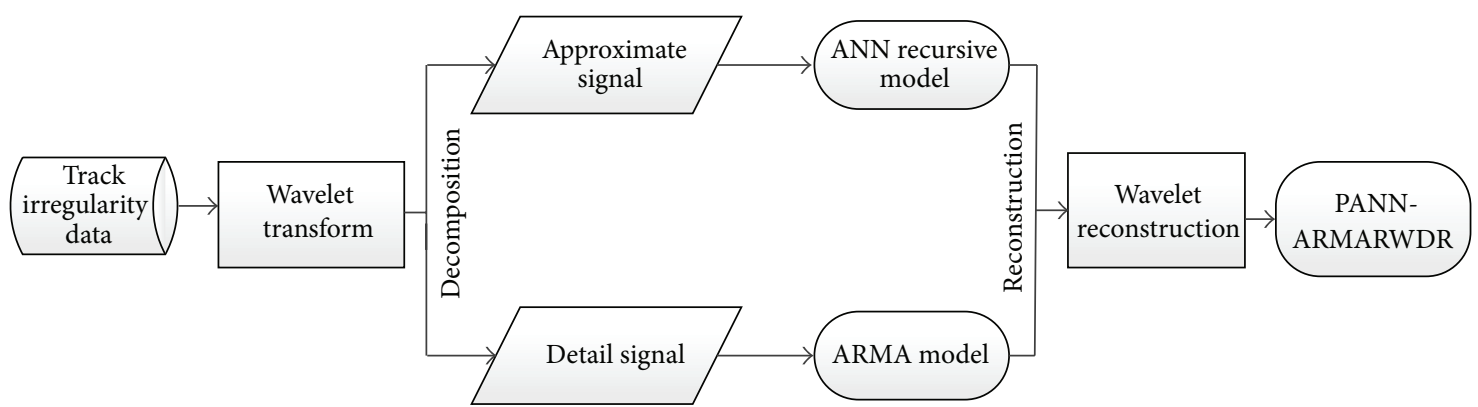

FIGURE 16: Modeling process of piecewise ANN-ARMA recursive model based on wavelet decomposition and reconstruction.

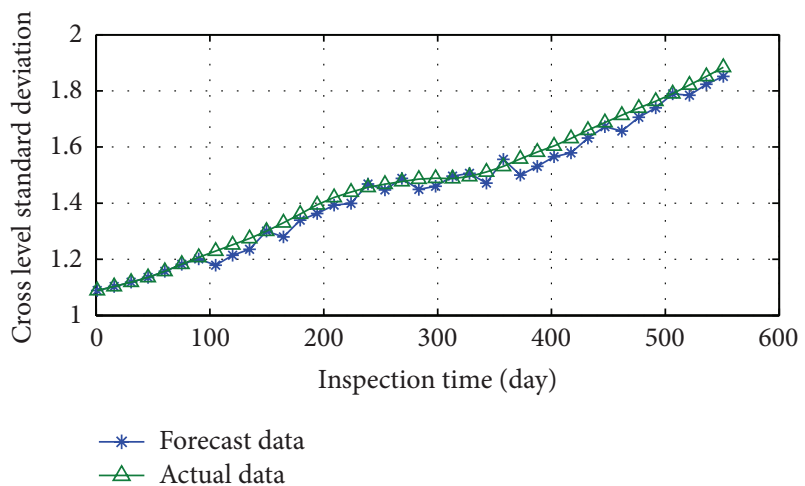

FIGURE 17: Forecast value and the original data of the third layer approximation waveform signal.

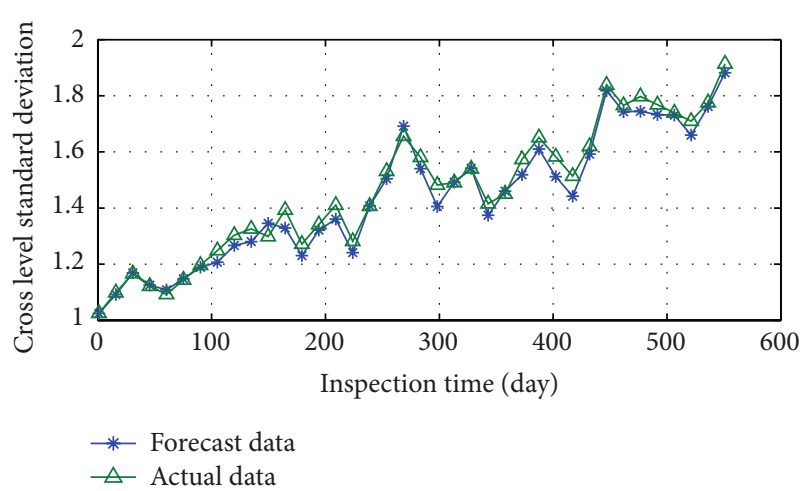

FIGURE 18: Comparison between forecast value and original value.

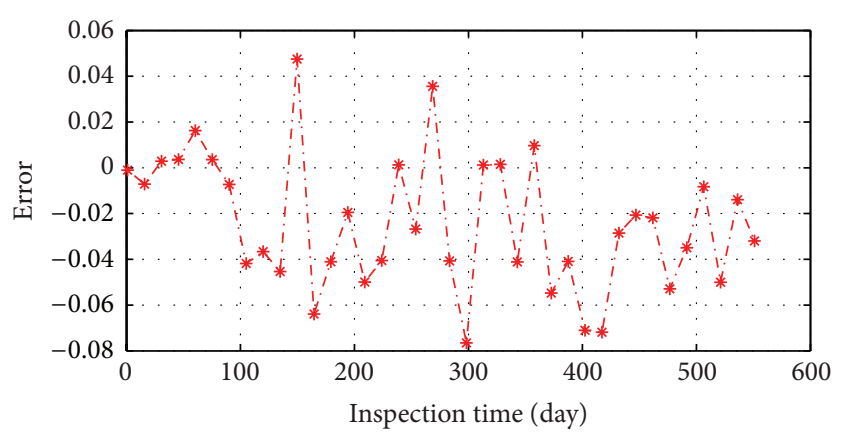

Figure 19: Error analysis.

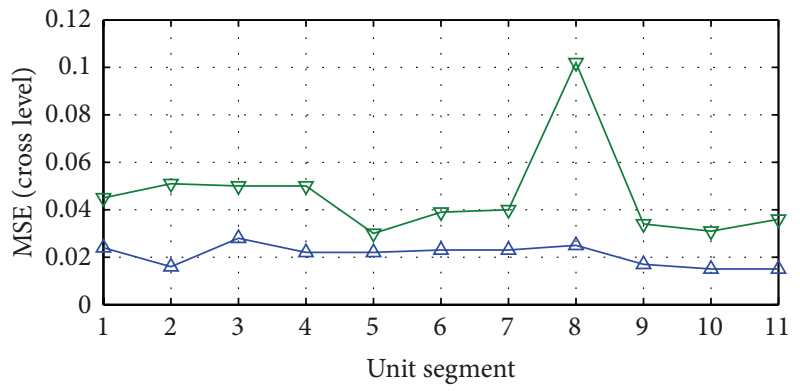

$\triangle$ PG-ARMARWDR

$\nabla$ PANN-ARMARWDR

Figure 20: Comparison of cross level MSE.

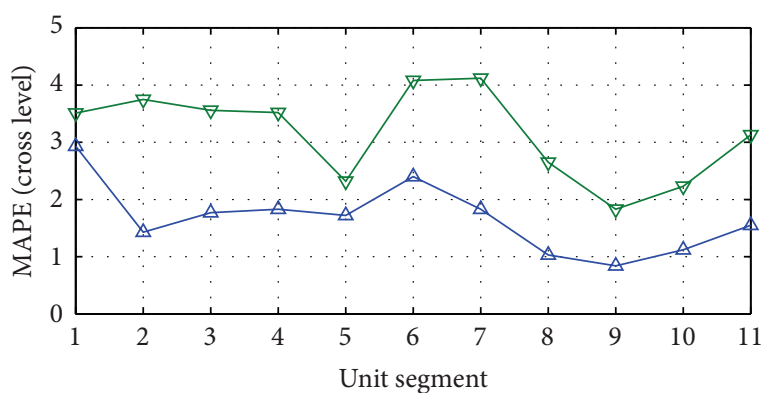

$\triangle$ PG-ARMARWDR

$\nabla$ PANN-ARMARWDR

Figure 21: Comparison of cross level MAPE.

node in the output layer is selected. $S$-function is selected as transfer function of the hidden layer; linear function is selected as transfer function of the output layer. Learning rate is set to be 0.1 , momentum coefficient is set to be 0.95 , and the maximum training time is set to be 30 seconds; variable learning rate momentum gradient descent algorithm (traingdx) is selected as the training algorithm, and minimum mean square error is set to be 0.001 . Through neural network estimation, approximation sequence estimation value $\widehat{A} 3^{(0)}$ can be obtained. High frequency detail sequence employs ARMA model to get the estimation values $\widehat{D} 1, \widehat{D} 2$, and $\widehat{D} 3$. Finally, the estimation model reconstructures the two results, 
TABLE 3: Analysis of model accuracy (cross level standard deviation).

\begin{tabular}{lcc}
\hline Unit section & MSE & MAPE \\
\hline K449+000-K449+025 & 0.045 & $3.51 \%$ \\
K449+100-K449+125 & 0.051 & $3.75 \%$ \\
K449+200-K449+225 & 0.050 & $3.56 \%$ \\
K449+325-K449+350 & 0.050 & $3.52 \%$ \\
K449+425-K449+450 & 0.030 & $2.32 \%$ \\
K449+475-K449+500 & 0.039 & $4.08 \%$ \\
K449+575-K449+600 & 0.040 & $4.12 \%$ \\
K449+675-K449+700 & 0.102 & $2.65 \%$ \\
K449+800-K449+825 & 0.034 & $1.83 \%$ \\
K449+875-K449+900 & 0.031 & $2.23 \%$ \\
K449+975-K450+000 & 0.036 & $3.13 \%$ \\
\hline
\end{tabular}

and all decomposed estimation sequence data are added up by weight 1 .

After "decomposition-estimation-reconstruction" process, the final forecast result is obtained, and the modeling process is shown in Figure 16.

5.3.2. Case Study of PANN-ARMARWDR. Piecewise ANN recursive model is used in low frequency approximation sequence signal of cross level irregularity standard deviation sequence data after wavelet decomposition and the forecast results are shown in Figure 17.

All forecast sequence data is added up by weight 1 , and the comparison of forecast values and original values is shown in Figure 18.

5.4. Accuracy and Error. Forecast error of the model is shown in Figure 19.

MSE and MAPE are used to measure accuracy of the model, and the forecast accuracy of some units is shown in Table 3 .

According to the forecast results of MSE, MAPE value in Table 3, as the model PG-ARMARWDR, PANNARMARWDR also has higher forecast accuracy. This has shown that PANN-ARMARWDR is also an effective way to forecast the trend of track state changes.

\section{Models Comparison}

This paper presents two forecast models based on wavelet decomposition-reconstruction, and both of them have achieved satisfactory forecasts. MSE and MAPE are used as forecast indicators. The comparison of the accuracy of the forecast models (PG-ARMARWDR and PANNARMARWDR) is shown in Figures 20 and 21.

MAPE forecast accuracy can be divided into four indicators: high accuracy forecast (MAPE $<10 \%$ ), sound forecast $(10 \%<$ MAPE < $20 \%)$, feasible forecast $(20 \%<$ MAPE < $50 \%$ ), and error forecast (MAPE $>50 \%$ ). According to MAPE indicator, both models proposed in this paper belong to high accuracy forecast. Meanwhile, it can be found from the comparison of the model's accuracy indicators MSE and MAPE from the chart that the forecast accuracy of PG-ARMARWDR model is higher than that of PANNARMARWDR model. Because of the sample size being small, the forecast accuracy of PANN-ARMARWDR is low, and so the forecast accuracy of PG-ARMARWDR is higher than PANN-ARMARWDR. If there is a large number of sample data, PANN-ARMARWDR accuracy will be improved significantly.

\section{Conclusions}

ARIMA model after residual modification has higher forecast accuracy than the original ARIMA model, but residual modification process not only increases the amount of computation model and complexity but also cannot reflect its changing nature. Through wavelet transform, the low frequency approximation sequence is more gentle and smooth, the trend is more obvious, and high frequency detail sequence is more stable. Based on this idea, the paper first proposes PG-ARMARWDR model. In the model, gray recursive model is used in the low frequency approximation recursive sequence, and ARMA model is used in high frequency detail sequence. Finally, the neural network model as an important nonlinear modeling method has been widely used and can approximate any nonlinear process, so this paper also proposes PANN-ARMARWDR model. In this model, neural network recursive model is used in the low frequency approximation sequences, and ARMA model is used in high frequency detail sequence. After the "decompositionmodeling-reconstruction" process, the model achieves an accurate forecast in the state of track irregularity change trend. Sample analysis shows that the model has achieved satisfactory results in short-term forecasting, and its MSE, MAPE indicators are within a higher accuracy range.

\section{Conflict of Interests}

The authors declare that they have no financial and personal relationships with other people or organizations that can inappropriately influence their work, and there is no professional or other personal interest of any nature or kind in any product, service, and/or company that could be construed as influencing the position presented in, or the review of, this paper.

\section{Acknowledgments}

This study was supported by the National Natural Science Foundation of China (Grant no.: 61272029) and National Key Technology R\&D Program (Grant no.: 2009BAG12A10).

\section{References}

[1] V. Genre, G. Kenny, A. Meyler, and A. Timmermann, "Combining expert forecasts: can anything beat the simple average?" International Journal of Forecasting, vol. 29, no. 1, pp. 108-121, 2013.

[2] J. S. Armstrong, "Combining forecasts," in Principles of Forecasting: A Handbook for Researchers and Practitioners, J. Scott 
Armstrong, Ed., pp. 417-439, Kluwer Academic Publishers, New York, NY, 2001.

[3] F.-M. Tseng, G.-H. Tzeng, H.-C. Yu, and B. J. Yuan, "Fuzzy ARIMA model for forecasting the foreign exchange market," Fuzzy Sets and Systems, vol. 118, no. 1, pp. 9-19, 2001.

[4] S.-M. Chen and N.-Y. Chung, "Forecasting enrollments using high-order fuzzy time series and genetic algorithms," International Journal of Intelligent Systems, vol. 21, no. 5, pp. 485-501, 2006.

[5] K. Huarng and T. H.-K. Yu, "The application of neural networks to forecast fuzzy time series," Physica A: Statistical Mechanics and Its Applications, vol. 363, no. 2, pp. 481-491, 2006.

[6] M. Khashei, S. R. Hejazi, and M. Bijari, "A new hybrid artificial neural networks and fuzzy regression model for time series forecasting," Fuzzy Sets and Systems, vol. 159, no. 7, pp. 769-786, 2008.

[7] S.-M. Chen, "Forecasting enrollments based on high-order fuzzy time series," Cybernetics and Systems, vol. 33, no. 1, pp. 116, 2002.

[8] H.-K. Yu, "Weighted fuzzy time series models for TAIEX forecasting," Physica A: Statistical Mechanics and Its Applications, vol. 349, no. 3-4, pp. 609-624, 2005.

[9] W. Y. Goh, C. P. Lim, and K. K. Peh, "Predicting drug dissolution profiles with an ensemble of boosted neural networks: a time series approach," IEEE Transactions on Neural Networks, vol. 14, no. 2, pp. 459-463, 2003.

[10] M. C. Medeiros and Á. Veiga, "A hybrid linear-neural model for time series forecasting," IEEE Transactions on Neural Networks, vol. 11, no. 6, pp. 1402-1412, 2000.

[11] G. Armano, M. Marchesi, and A. Murru, "A hybrid geneticneural architecture for stock indexes forecasting," Information Sciences, vol. 170, no. 1, pp. 3-33, 2005.

[12] P. G. Zhang, "Time series forecasting using a hybrid ARIMA and neural network model," Neurocomputing, vol. 50, pp. 159175, 2003.

[13] F.-M. Tseng, H.-C. Yu, and G.-H. Tzeng, "Combining neural network model with seasonal time series ARIMA model," Technological Forecasting and Social Change, vol. 69, no. 1, pp. 71-87, 2002.

[14] D. S. K. Karunasinghe and S.-Y. Liong, "Chaotic time series prediction with a global model: artificial neural network," Journal of Hydrology, vol. 323, no. 1-4, pp. 92-105, 2006.

[15] M. J. Aitkenhead, A. J. S. McDonald, J. J. Dawson et al., “A novel method for training neural networks for time-series prediction in environmental systems," Ecological Modelling, vol. 162, no. 1, pp. 87-95, 2003.

[16] S. BuHamra, N. Smaoui, and M. Gabr, "The Box-Jenkins analysis and neural networks: prediction and time series modelling," Applied Mathematical Modelling, vol. 27, no. 10, pp. 805-815, 2003.

[17] H. Niska, T. Hiltunen, A. Karppinen, J. Ruuskanen, and M. Kolehmainen, "Evolving the neural network model for forecasting air pollution time series," Engineering Applications of Artificial Intelligence, vol. 17, no. 2, pp. 159-167, 2004.

[18] G. P. Zhang and M. Qi, "Neural network forecasting for seasonal and trend time series," European Journal of Operational Research, vol. 160, no. 2, pp. 501-514, 2005.

[19] K.-J. Kim, "Financial time series forecasting using support vector machines," Neurocomputing, vol. 55, no. 1-2, pp. 307-319, 2003.
[20] G. P. Zhang, "Time series forecasting using a hybrid ARIMA and neural network model," Neurocomputing, vol. 50, pp. 159175, 2003.

[21] H. Ince and T. B. Trafalis, "A hybrid model for exchange rate prediction," Decision Support Systems, vol. 42, no. 2, pp. 10541062, 2006.

[22] P.-C. Chang, C.-H. Liu, and Y.-W. Wang, "A hybrid model by clustering and evolving fuzzy rules for sales decision supports in printed circuit board industry," Decision Support Systems, vol. 42, no. 3, pp. 1254-1269, 2006.

[23] K. Huarng and T. H.-K. Yu, "The application of neural networks to forecast fuzzy time series," Physica A: Statistical Mechanics and its Applications, vol. 363, no. 2, pp. 481-491, 2006.

[24] X. Deng and X. Wang, "Incremental learning of dynamic fuzzy neural networks for accurate system modeling," Fuzzy Sets and Systems, vol. 160, no. 7, pp. 972-987, 2009.

[25] P.-F. Pai and C.-S. Lin, "A hybrid ARIMA and support vector machines model in stock price forecasting," Omega, vol. 33, no. 6, pp. 497-505, 2005.

[26] K.-Y. Chen and C.-H. Wang, "A hybrid SARIMA and support vector machines in forecasting the production values of the machinery industry in Taiwan," Expert Systems with Applications, vol. 32, no. 1, pp. 254-264, 2007.

[27] Z.-J. Zhou and C.-H. Hu, "An effective hybrid approach based on grey and ARMA for forecasting gyro drift," Chaos, Solitons \& Fractals, vol. 35, no. 3, pp. 525-529, 2008.

[28] L. Yu, S. Wang, and K. K. Lai, "A novel nonlinear ensemble forecasting model incorporating GLAR and ANN for foreign exchange rates," Computers \& Operations Research, vol. 32, no. 10, pp. 2523-2541, 2005.

[29] H.-J. Kim and K.-S. Shin, "A hybrid approach based on neural networks and genetic algorithms for detecting temporal patterns in stock markets," Applied Soft Computing Journal, vol. 7, no. 2, pp. 569-576, 2007.

[30] M. Khashei, M. Bijari, and G. A. Raissi Ardali, "Improvement of auto-regressive integrated moving average models using fuzzy logic and Artificial Neural Networks (ANNs)," Neurocomputing, vol. 72, no. 4-6, pp. 956-967, 2009.

[31] H. F. Lotfi, R. G. Jahanshahloo, S. Givehchi, and M. VaezGhasemi, "Using DEA-neural network approach to solve binary classification problems," Journal of Data Envelopment Analysis and Decision Science, vol. 2013, Article ID dea-00002, 12 pages, 2013.

[32] N. V. Karpenka, F. Feroz, and M. P. Hobson, "A simple and robust method for automated photometric classification of supernovae using neural networks," Monthly Notices of the Royal Astronomical Society, vol. 429, no. 2, pp. 1278-1285, 2013.

[33] H. A. Mallot, Artificial Neural Networks, Computational Neuroscience, Springer, New York, NY, USA, 2013.

[34] N. Gupta, "Artificial neural network," Network and Complex Systems, vol. 3, no. 1, pp. 24-28, 2013.

[35] K. S. Kasiviswanathan and K. P. Sudheer, "Quantification of the predictive uncertainty of artificial neural network based river flow forecast models," Stochastic Environmental Research and Risk Assessment, vol. 27, no. 1, pp. 137-146, 2013.

[36] M. R. N. Avanaki, P. P. Laissue, T. J. Eom, A. G. Podoleanu, and A. Hojjatoleslami, "Speckle reduction using an artificial neural network algorithm," Applied Optics, vol. 52, no. 21, pp. 50505057, 2013.

[37] J. Hashimoto, K. Ogawa, J. Bai, A. Kubo, and Y. Imai, "Simultaneous dual-isotope imaging based on an artificial neural 
network for evaluating myocardial perfusion and fatty acid metabolism," Journal of Nuclear Cardiology, vol. 20, no. 3, pp. 396-405, 2013.

[38] S. Pascale, S. Parisi, A. Mancini et al., "Landslide susceptibility mapping using artificial neural network in the Urban area of Senise and San Costantino Albanese (Basilicata, Southern Italy)," in Computational Science and Its Applications-ICCSA 2013, vol. 7974 of Lecture Notes in Computer Science, pp. 473488, Springer, Berlin, Germany, 2013.

[39] M.-T. Wu and Y. Yong, "The research on stock price forecast model based on data mining of BP neural networks," in Proceedings of the 3rd IEEE International Conference on Intelligent System Design and Engineering Applications (ISDEA '13), pp. 1526-1529, January 2013.

[40] D. Carlucci, P. Renna, and G. Schiuma, "Evaluating service quality dimensions as antecedents to outpatient satisfaction using back propagation neural network," Health Care Management Science, vol. 16, no. 1, pp. 37-44, 2013.

[41] M. Buscema, "Supervised artificial neural networks: backpropagation neural networks," in Intelligent Data Mining in Law Enforcement Analytics, pp. 119-135, Springer, Amsterdam, The Netherlands, 2013. 


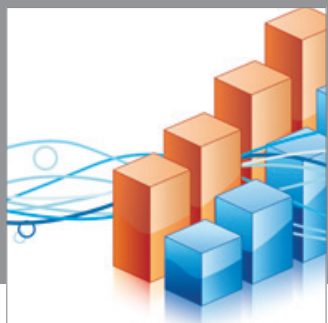

Advances in

Operations Research

mansans

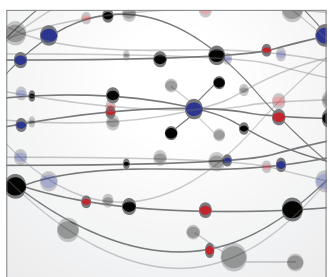

The Scientific World Journal
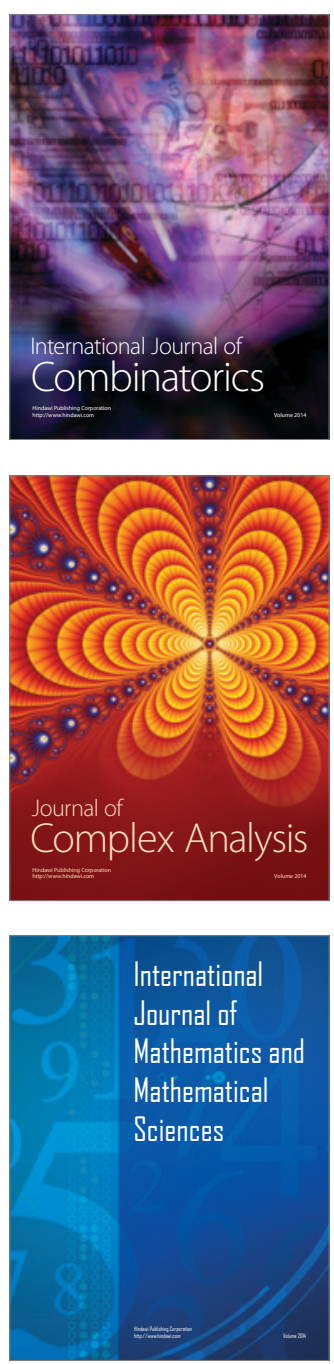
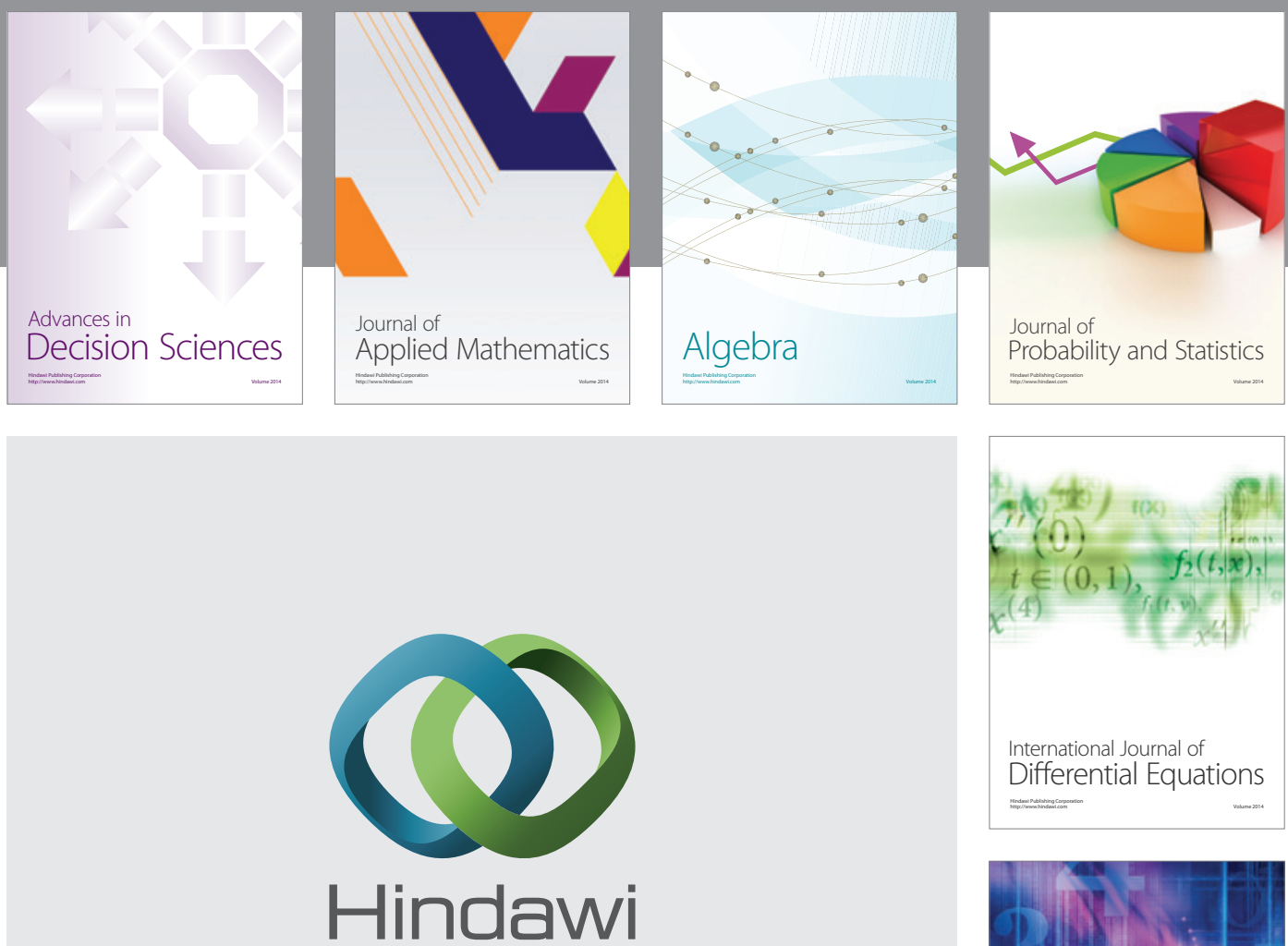

Submit your manuscripts at http://www.hindawi.com
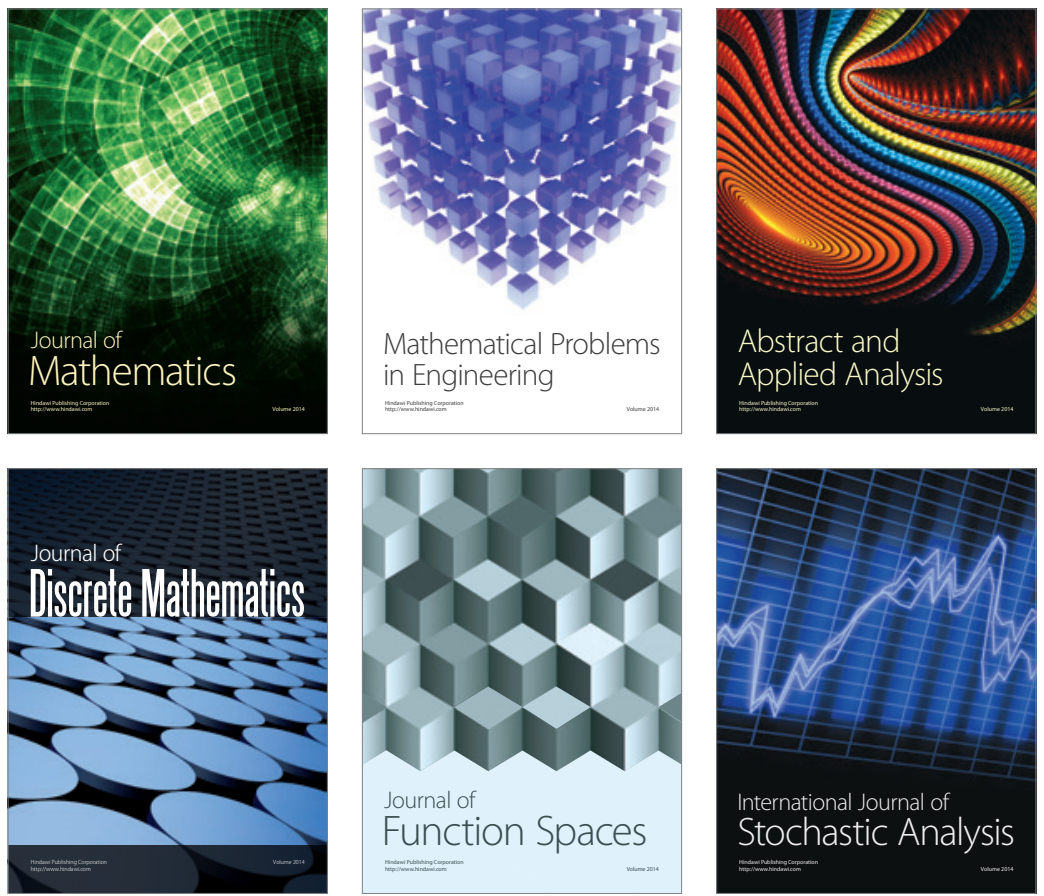

Journal of

Function Spaces

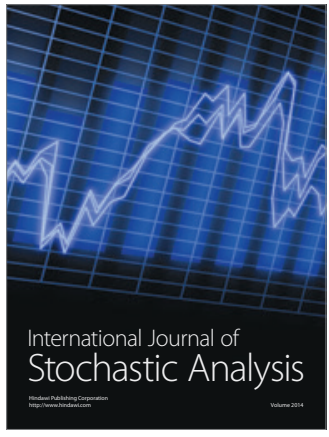

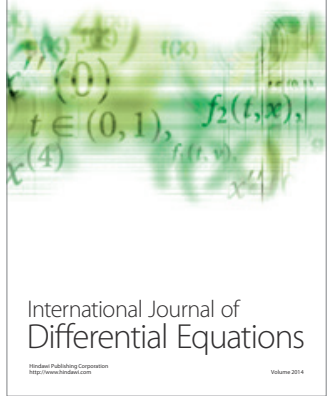
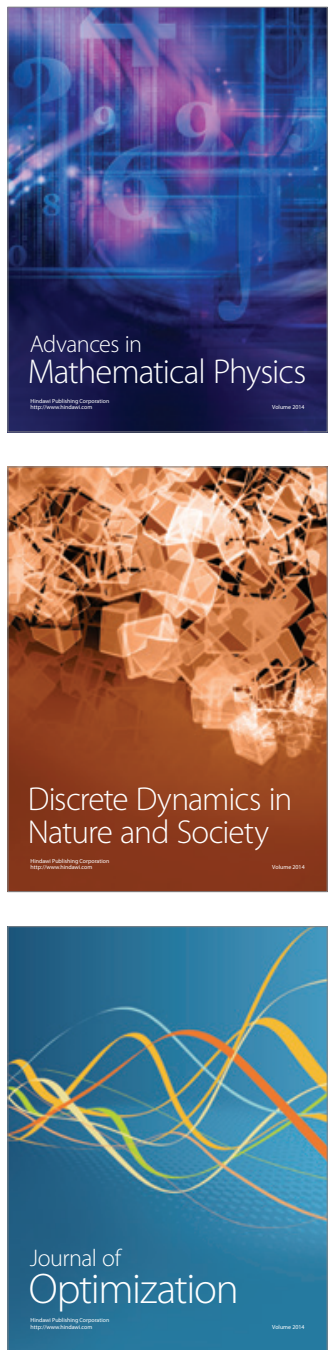